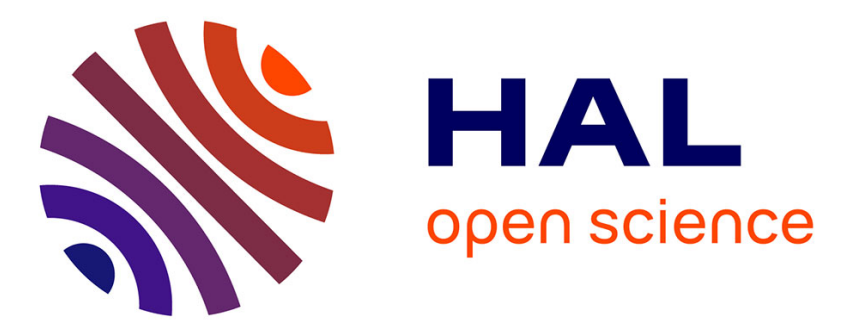

\title{
Les pratiques de développement durable et de RSE en région PACA : la spécificité des petites entreprises en question
}

Véronique Bon, Alain Lacroux, Pierre Teller, Corinne van Der Yeught

\section{- To cite this version:}

Véronique Bon, Alain Lacroux, Pierre Teller, Corinne van Der Yeught. Les pratiques de développement durable et de RSE en région PACA: la spécificité des petites entreprises en question. Colloque "gouvernance et création de valeur(s)", Apr 2012, Toulon, France. hal-00689826

\section{HAL Id: hal-00689826 https://hal.science/hal-00689826}

Submitted on 20 Apr 2012

HAL is a multi-disciplinary open access archive for the deposit and dissemination of scientific research documents, whether they are published or not. The documents may come from teaching and research institutions in France or abroad, or from public or private research centers.
L'archive ouverte pluridisciplinaire HAL, est destinée au dépôt et à la diffusion de documents scientifiques de niveau recherche, publiés ou non, émanant des établissements d'enseignement et de recherche français ou étrangers, des laboratoires publics ou privés. 


\title{
Les pratiques de développement durable et de RSE en région PACA : la spécificité des petites entreprises en question
}

\author{
Colloque « Gouvernance et création de valeur(s) » \\ 6èmes Journées scientifiques de l'Université du Sud Toulon-Var
}

Toulon le 17 avril 2012

Auteurs :

Véronique Bon

Maître de conférences

IAE, Université du Sud Toulon-Var

Groupe de Recherche en Management (GRM, EA 4711)

Alain Lacroux,

Maître de conférences

IUT de Draguignan, Université du Sud Toulon-Var

Groupe de Recherche en Management (GRM, EA 4711)

Pierre Teller

Maître de conférences

IAE, Université de Nice Sophia Antipolis

Groupe de Recherche en Management (GRM, EA 4711)

Corinne Van Der Yeught

Maître de conférences

IAE, Université du Sud Toulon-Var

Groupe de Recherche en Management (GRM, EA 4711) 


\section{Les pratiques de développement durable et de RSE en région PACA : la spécificité des petites entreprises en question}

\section{RESUME :}

Alors que la norme mondiale ISO 26000 , votée fin 2010, consacre l'adossement de la responsabilité sociétale des organisations à l'objectif du développement durable de la planète, les petites entreprises, par ailleurs peu représentées dans le processus d'élaboration de la norme, sont-elles en mesure de répondre à l'exigence d'une responsabilité adossée à cet objectif ? Portent-elles des spécificités en la matière ? Si la spécificité des PME a fait l'objet de multiples travaux, en particulier dans le champ de la responsabilité sociétale, le rapport des PME au développement durable (perceptions et pratiques) est essentiellement interrogé par le biais d'études qualitatives sans que l'hypothèse d'une éventuelle spécificité soit testée. Cette étude s'inscrit donc dans cet espace encore peu exploré par les méthodologies quantitatives. Réalisée auprès de 156 organisations de la région PACA dont 129 entreprises, elle conduit à des résultats contrastés où l'hypothèse d'une spécificité des PE concernant leurs pratiques en RSE et DD est partiellement validée.

Mots clés : RSE, développement durable, petites entreprises, région PACA

The ISO 26000 standard, which has been applicable worldwide since 2010, puts into light the link between corporate social responsibility and global sustainable development. Nevertheless, it is doubtful that small businesses, which, incidentally, have been poorly involved in the standard's elaboration process, are in a position to face demands based on such ambitious objectives as corporate social responsibility and sustainable development. It may be suggested, on the contrary, that small businesses should be regarded as a case apart. Admittedly, a lot of research has hitherto focused on the specificities of small businesses in the field of corporate social responsibility. However, the link between small businesses and sustainable development (as regards both perception and practice) has generally been addressed through qualitative studies that rarely consider they that feature specificities of their own. This study tries to explore this peculiarity by using quantitative methodologies and it is based on data collected from 156 organizations in the Provence-Alpes-Côte d'Azur area (in southeastern France). It reaches contrasting results but partially validates the hypothesis of a specific practice of corporate social responsibility and sustainable development in small businesses.

Key words : CSR, sustainability, small businesses, PACA area 
Introduction

Alors que la norme mondiale ISO 26000 , votée fin 2010, consacre l'adossement de la responsabilité sociétale des organisations (RSE) à l'objectif du développement durable (DD) de la planète, les petites entreprises, par ailleurs peu représentées dans le processus d'élaboration de la norme, sont-elles en mesure de répondre à cette exigence de responsabilité ? Portent-elles des spécificités susceptibles de favoriser ou de contrarier un tel engagement?

Si la spécificité de «l'idéal-type PME » a fait l'objet de multiples travaux, en particulier dans le champ de la responsabilité sociétale, le rapport des PME au développement durable (perceptions et pratiques) est essentiellement interrogé par le biais d'études qualitatives sans que l'hypothèse d'une éventuelle spécificité ait été soumise au test statistique. Notre étude s'inscrit dans cet espace encore peu exploré par les méthodologies quantitatives. Au plan méthodologique, nous avons tout d'abord constitué une base de données réunissant à ce jour 156 organisations, parmi lesquelles 129 entreprises de la région PACA. Puis, nous avons élaboré un questionnaire qui a été administré en face à face. L'information collectée est entièrement consacrée aux démarches en RSE et en management du développement durable, qu'il s'agisse d'appréhender les facteurs d'engagement des organisations, les freins et les avantages organisationnels perçus ou encore le degré de connaissance des répondants (dirigeants d'entreprise ou responsables). Dans le cadre de notre question de recherche, les données recueillies ont fait l'objet de tests systématiques visant à comparer les petites entreprises (PE, moins de 50 salariés) et leurs homologues de plus grande taille (Moyennes et Grandes entreprises, MGE). La petite entreprise représente la structure organisationnelle la plus à même de représenter «l'idéal-type PME » décrit par les chercheurs (Spence, 2007).

La première partie de cette contribution aborde la façon dont la littérature en Sciences de gestion appréhende la spécificité de ces organisations et s'intéresse plus particulièrement aux travaux en RSE/DD inscrits dans un champ que Torrès qualifie de "paradigme de la spécificité ». Cette revue de littérature fait émerger les questionnements guidant la recherche quantitative engagée.

En seconde partie, en sus d'une présentation de la méthodologie retenue, une première étude met en évidence les traits saillants de notre échantillon global au regard des champs de questionnements retenus. La troisième et dernière partie est consacrée à l'exposé tant des spécificités identifiées au sein des petites entreprises que des traits (perceptions et pratiques) qu'elles partagent avec leurs homologues de plus grande taille. A l'évidence, les résultats statistiques conduisent à relativiser les présomptions établies à partir de la littérature, même si certains traits spécifiques sont identifiés ou confirmés. En conclusion, nous discutons la portée et les limites de ces résultats.

\section{Le questionnement d'une spécificité des petites entreprises en RSE/DD}

Si la recherche en PME s'inscrit de façon dominante dans le paradigme de la spécificité, il est nécessaire ici d'interroger ce cadre de référence, d'en définir les traits éventuels. Ce paradigme n'est en effet acceptable qu'à partir du moment où il est soumis à un examen critique comme toute hypothèse réfutable (Torrès, 1998). Les traits de cette spécificité caractérisent la PME en tant que concept ou encore idéal-type (Julien et Marchenay, 1988). A la petite taille des effectifs et(ou) du chiffre d'affaires, s'ajoutent une gestion centralisée dans les mains d'une équipe de direction réduite, une personnalisation de la gestion autour de la personne du dirigeant-propriétaire, un système d'information (interne ou externe) simple et peu formalisé, une spécialisation réduite des tâches et un processus de décision stratégique empruntant largement à l'intuition et au bon sens, sans planification formelle.

Torres (2000) définit cette spécificité au travers d'une proximité géographique, affective et cognitive du dirigeant-propriétaire vis-à-vis de ses partenaires et collaborateurs. Courrent et 
Torrès (2005) soulignent l'ambivalence de ces proxémies, synonyme de liens forts vis-à-vis des partenaires les plus proches mais également d'exclusion de parties prenantes éloignées.

Cette ambivalence se traduit par un management spécifique des parties prenantes: les partenaires proches (couramment, les clients, les employés, les fournisseurs) font l'objet d'une attention managériale forte mais les attentes de la société ou des pouvoirs publics sont relayées au rang de préoccupations secondaires (voir la revue de littérature de Lepoutre et Heene, 2006). Jenkins (2004) relève ainsi une moindre sensibilité des dirigeants de PME aux pressions de l'Etat, des collectivités locales et des groupes d'intérêt.

Les chercheurs ont précisément interrogé cette spécificité de la PME-concept au regard de sa propension et de sa capacité à engager des démarches socialement responsables. Ils soulignent l'importance accordée aux relations interpersonnelles, lesquelles favorisent la compréhension et la prise en compte des attentes des partenaires concernés (Spence, 1999 ; Bon, 2007), une approche dialectique associée à la recherche d'un consensus social (Paradas, 2008) et la constitution d'un capital social (Spence et al., 2003 ; Russo et Perrini, 2010). Non soumis à la pression à court terme d'un actionnariat attaché à la maximisation des profits, le dirigeantpropriétaire dispose d'une marge de manœuvre supplémentaire pour engager une politique socialement responsable (Spence, 2007 ; Jenkins, 2004). Le système de gouvernance est en ce sens jugé plus propice à la mise en acte d'une éthique managériale de la responsabilité sociétale (Bon et Morlet, 2011). Les valeurs des dirigeants jouent un rôle clé pour expliquer l'engagement socialement responsable de ces entreprises (Murillo et Lozano, 2006; Quinn, 1997). La souplesse et la flexibilité organisationnelle facilitent la diffusion et la promotion en interne de ces valeurs (Fassin, Van Rossem et Buelens, 2011). Conjointement, les chercheurs pointent du doigt les freins à la mise en place de politiques en responsabilité sociétale : une latitude réduite au plan financier, un dirigeant multifonctions accaparé par la gestion quotidienne de l'entreprise et des ressources humaines limitées en particulier quand il s'agit de structurer ces démarches ou de les instrumentaliser (Spence, 1999; Jenkins, 2004).

Ces travaux explorant les pratiques des PME en RSE et leur mode de management des parties prenantes fournissent un éclairage intéressant pour appréhender au plan théorique la question des pratiques en matière de management du développement durable. Le développement durable a reçu une définition officielle en 1987 dans un rapport onusien intitulé «Our common future ». Il invite les Etats à engager un mode de "développement qui réponde aux besoins du présent sans compromettre la capacité des générations futures de répondre aux leurs ». Originellement adressée aux Etats, l'exigence de durabilité est étendue aux organisations et en particulier aux entreprises lors des sommets de la Terre organisés à Rio en 1992 et à Johannesburg en 2002. Les enjeux de préservation environnementale, initialement dominants dans l'appréhension du développement durable (l'éco-développement, mis à l'honneur lors du sommet de Stockholm de 1972 (Sachs, 1980, 1997)), laissent alors place à une approche plus ouverte où il s'agit d'assurer un développement économique équitable dans un environnement viable et vivable. L'entreprise a la responsabilité d'engager son activité économique dans le respect de cet attendu, impliquant à la fois la prise en compte des parties prenantes contractuelles, des parties muettes comme l'environnement naturel et des parties absentes telles que les futures générations. Le cadre normatif mondial établissant «les lignes directrices de la responsabilité sociétale » ISO 26 000, texte voté fin 2010 à l'issue d'un processus international d'élaboration multipartite, adosse ainsi la responsabilité des organisations aux objectifs du développement durable (Bon, 2011).

$\mathrm{Au}$ regard des spécificités présumées de l'idéal-type PME au plan de sa prise de responsabilité sociétale, dans quelle mesure ces entreprises appréhendent-elles et prennentelles en compte les exigences du développement durable dans leurs pratiques ? Certes, leur flexibilité et leur souplesse organisationnelles favorisent les stratégies de rupture et les politiques d'innovation radicale permettant d'explorer les voies nouvelles portées par 
l'objectif du développement durable (Moore et Manring, 2009 ; Bocquet et Mothe, 2010). Mais, l'égocentrisme présumé du dirigeant ne comporte-t-il pas le risque de circonscrire les préoccupations managériales aux parties prenantes les plus proches (les partenaires contractuels notamment), laissant de côté les enjeux portés par des groupes auxquels le dirigeant est finalement moins sensible ? L'impact environnemental et communautaire des activités économiques mobiliserait alors une moindre attention managériale tandis que les proxémies et l'importance accordée aux relations interpersonnelles laisseraient augurer d'une attention plus soutenue aux partenaires concernés (en particulier, les collaborateurs) et finalement d'une assomption plus profonde des responsabilités en la matière. Cette hypothèse suscite une série de questionnements : la portée macroéconomique du développement durable permet-elle à ce dirigeant d'envisager la pertinence d'une contribution organisationnelle à cet objectif planétaire ? Quand l'engagement de telles démarches est avéré, est-il impulsé sous la pression de parties prenantes ou instrumentalisé dans le cadre d'une stratégie d'entreprise ? Des motivations éthiques ou morales du dirigeant interviennent-elles plus particulièrement dans la décision d'engagement ? Cet engagement est-il effectivement contrarié par des ressources financières, temporelles, humaines et cognitives jugées limitées, comme le suppose généralement la littérature ? Quelle connaissance le dirigeant a-t-il du développement durable et des pratiques managériales associées ? Le système d'information dessine-t-il des pratiques spécifiques en matière de politiques $\mathrm{DD}$, en particulier au plan des dispositifs et instruments de gestion mobilisés?

La grande majorité des études scientifiques conduites sur le thème des politiques en développement durable/RSE au sein des petites entreprises en Europe mobilise des études qualitatives donnant à voir des processus d'engagement bien souvent exemplaires (par exemple, Jenkins, 2009 ; Murillo et al., 2006). Si les facteurs clés de succès de ces démarches et les processus d'engagement sont appréhendés finement, ces études ne permettent pas d'inférer scientifiquement une spécificité. Les cas retenus sont rarement comparés à leurs homologues de plus grande taille et rendent compte d'études monographiques adaptées à ces approches en profondeur (par exemple, Paradas, 2008). Quand cette comparaison est envisagée (Dupuis et al., 2006; Berger-Douce, 2008), l'approche quantitative n'est généralement pas assortie de tests statistiques. Quelques études disponibles au niveau français sont également proposées par des organisations professionnelles, en particulier les chambres consulaires (le baromètre annuel du Crocis-CCI ou l'étude de l'ACFCI, 2006). Néanmoins, elles distinguent les grandes entreprises et les PME, en retenant des seuils d'effectifs salariés et des périmètres d'étude différenciés. ${ }^{1}$

Questionnant la spécificité de l'idéal-type PME, nous prenons le parti d'envisager les démarches des entreprises de moins de 50 salariés relativement aux entreprises de taille supérieure, suivant en cela les recommandations de Spence (2007). En effet, les moyennes entreprises (de 50 à 250 salariés) présentent des caractéristiques organisationnelles hybrides les rapprochant de la grande entreprise: un système d'information plus formalisé, des " proxémies » moins évidentes associées à des marchés élargis et(ou) à des parties prenantes plus éloignées (Bon, 2007). Ces entreprises sont également plus fréquemment soumises aux phénomènes de dénaturation susceptibles d'infléchir les spécificités présumées, notamment quand leur croissance s'accompagne d'une perte d'indépendance capitalistique (Le Vigoureux, 2003).

\footnotetext{
${ }^{1}$ L'étude de l'ACFCI retient un échantillon de 100 entreprises de la région parisienne ayant engagé depuis plus de 6 mois des démarches en DD. 92\% des entreprises sondées par questionnaire ont moins de 250 salariés (seuil PME retenu par l'étude). Le Crocis-CCI publie depuis 2006 un «baromètre du développement durable », établi à partir d'un entretien téléphonique auprès de plus de 5000 dirigeants d'entreprises de la région parisienne, en opérant une distinction des PME et des grandes entreprises sur la base du seuil de 500 salariés.
} 


\section{De la méthodologie de recherche à l'échantillon constitué}

Pour mener à bien cette étude, nous avons utilisé une base de données constituée dans le cadre d'un projet de recherche mené par un collectif d'enseignants-chercheurs de notre laboratoire. Ce projet s'appuie sur la collecte d'informations auprès des organisations de la région PACA via l'élaboration d'un questionnaire interrogeant leurs pratiques et perceptions en matière de développement durable. Après une phase de pré-test de quinze entreprises, ce questionnaire a été administré en face à face par les étudiants de nos formations respectives (Licences et Master en management) pendant une période d'un an et demi environ (2009-2010). La base de données réunit à ce jour 156 questionnaires concernant des organisations de la région PACA dont précisément, 129 entreprises. L'échantillon se compose d'organisations appartenant à différents secteurs d'activité : la moitié dans le secteur des services, un tiers environ dans le commerce et un peu plus de $10 \%$ dans l'industrie et le BTP. Les petites et très petites organisations représentent $71 \%$ des observations. Ce questionnaire d'une quinzaine de pages aborde de nombreuses thématiques intéressant directement notre hypothèse de recherche. ${ }^{2}$ Les répondants (dirigeants et responsables de services) sont ainsi invités à se prononcer sur une série de questions dont les principales sont synthétisées ciaprès. Les résultats obtenus à partir de l'échantillon complet (toutes organisations) sont évoqués en distinguant trois domaines d'investigation :

- le degré de connaissance du répondant, la contribution perçue de son organisation au DD et l'engagement effectif en DD de son organisation,

- l'importance perçue des enjeux sociaux et environnementaux pour l'organisation,

- les freins, les avantages et les facteurs moteurs de l'engagement en DD de son organisation.

A chaque domaine exploré, correspondent des questions fermées permettant des réponses sous forme d'échelles ordinales ou d'échelles d'intervalles. Ces questionnements sont examinés à l'aune de l'hypothèse d'une spécificité des petites entreprises (moins de 50 salariés) en troisième partie.

\section{a. La connaissance du DD, la contribution perçue au DD, l'engagement effectif en DD}

Notre échantillon global indique que le DD est considéré par la grande majorité des répondants comme un enjeu citoyen important ; 93,5\% agissent dans leur vie quotidienne en faveur du DD et(ou) considèrent que le $\mathrm{DD}$ est une nécessité à prendre en considération sérieusement. Seulement, $6.5 \%$ des répondants l'appréhendent comme une démarche accessoire ne constituant pas leur priorité ; un unique répondant se dit non concerné en tant que citoyen. Cette observation révèle un objectif largement admis et partagé. Notons que le traitement des questions environnementales constitue pour les personnes sensibilisées le domaine le plus important, bien avant le domaine social. Dans l'esprit des répondants, le concept du DD est donc relié en premier lieu à l'axe environnemental. La primauté accordée aux enjeux environnementaux découle à la fois d'un processus originel d'émergence du concept centré sur ces questions (Steurer et al., 2005) et d'une médiatisation encore largement orientée dans ce sens.

Cela étant, la notoriété du développement durable auprès des répondants ne préjuge pas de leur degré de connaissance. $6 \%$ des répondants déclarent n'avoir aucune connaissance sur le sujet et ils sont $63 \%$ à disposer de connaissances se limitant à quelques domaines ; 12\% seraient en mesure d'en donner une définition précise. Enfin, alors que le répondant en tant

\footnotetext{
2 Le questionnaire tel qu'il a été remis aux répondants est disponible à l'adresse web suivante : http://www.tinyurl.com/3mofue6. Tous les membres du collectif qui ont contribué à l'élaboration du questionnaire jusqu'à la collecte de données sont cités.
} 
que citoyen admet l'importance du développement durable, il est plus mesuré quant à la capacité de son organisation à contribuer à cet objectif. Quel que soit le domaine, une proportion significative de répondants (entre $29.5 \%$ et $36 \%$ selon le domaine) considère que la capacité de l'entreprise à contribuer au DD est difficile ou très difficile.

\section{b. L'importance perçue des enjeux sociaux et environnementaux pour l'organisation}

En matière sociale, les enjeux jugés les plus importants recouvrent peu ou prou les thèmes traditionnels des politiques de gestion des ressources humaines : la santé et la sécurité, l'amélioration des conditions de travail, la formation professionnelle. Les autres enjeux comme l'équilibre vie privée/vie professionnelle ou encore les horaires choisis ou la participation des salariés aux décisions sont jugés moins importants. Il se dessine donc une hiérarchisation des enjeux plutôt au profit des champs d'action traditionnels de la GRH.

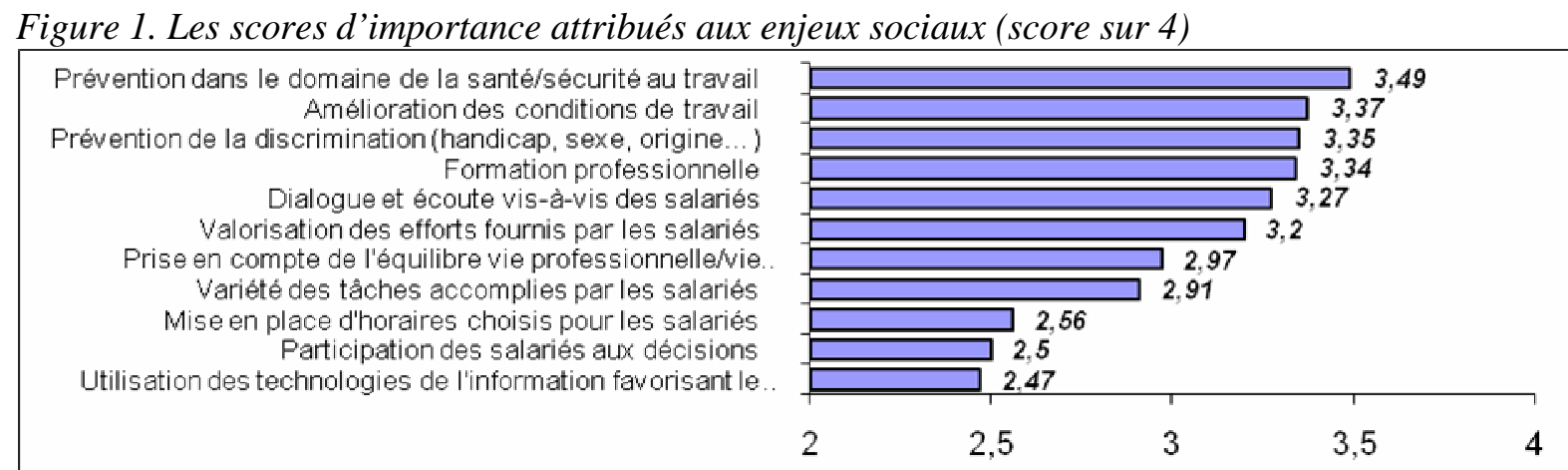

Concernant les enjeux environnementaux, le score moyen d'importance pour chaque item est supérieur à 3 (score sur 4). Même si la gestion des déchets constitue l'enjeu le plus important, il n'apparaît pas de hiérarchisation nette. Cela étant, $17 \%$ des répondants déclarent manquer d'informations sur l'impact environnemental de leur activité (contre 9\% pour l'impact social). Et pour $21 \%$ d'entre eux, la question ne se pose pas dans leur organisation. Autrement dit, un répondant sur 5 n'appréhende pas les questions environnementales sous cet angle.

\section{c. Les freins, les avantages et les facteurs moteurs de l'engagement en DD}

Selon les répondants, une démarche en DD permet d'abord de répondre à une exigence éthique externe, qu'elle soit codifiée par la loi (réglementation) ou portée par la société. Une telle démarche est également susceptible d'améliorer la réputation de l'entreprise. Les avantages de marché sont reconnus mais de façon moins nette. L'opportunité d'une réduction des coûts constitue un avantage peu mis en avant; il en est de même de la rentabilité financière. Les répondants dont les organisations ont déjà engagé des démarches DD, mettent en avant, parmi les facteurs favorisant leur engagement, des attentes d'ordre éthique, qu'elles soient inhérentes à l'individu lui-même (un devoir moral envers le citoyen), promues par son organisation par le biais des valeurs, codifiées par la réglementation ou encore portées par les partenaires publics. Les attentes des partenaires commerciaux et financiers jouent un rôle plus mesuré, voire limité dans les ressorts de cet engagement. Les facteurs cités sont représentatifs d'une institutionnalisation du développement durable qui s'est, au départ, construite en marge du monde économique. Quant aux facteurs susceptibles de freiner l'engagement en DD, la méconnaissance des instruments du DD arrive en tête, indiquant que l'instrumentation de gestion est considérée par les répondants comme un point d'entrée important dans une démarche DD. Notons que l'instrumentation, vecteur de rationalisation (Moisdon, 1997), est un réducteur de complexité opportun dans un domaine où les répondants se disent plutôt mal informés (3ième frein d'engagement cité). L'insuffisance de moyens financiers et une 
conjoncture économique défavorable sont également considérées comme des obstacles au déploiement d'une démarche DD. Il est intéressant de noter que l'impact sur la rentabilité financière n'est considéré ni comme un frein, ni comme un avantage significatif. Au plan cognitif, les répondants n'établissent pas de lien fort entre la performance financière et les enjeux du développement durable.

L'ensemble des résultats évoqués sont-ils différenciés quand il s'agit des petites entreprises (moins de 50 salariés) ? Dans quelle mesure révèlent-ils des spécificités propres aux PE? Nous avons soumis les questions explorées à une série de tests de comparaison entre PE et MGE, utilisant essentiellement le test paramétrique de Fischer (test F) et le test non paramétrique de Mann-Whitney (test MW). ${ }^{3}$

\section{La spécificité des petites entreprises en RSE/DD : des résultats nuancés}

L'échantillon retenu montre à l'évidence que la contribution de l'entreprise au DD est plus difficile à appréhender pour le dirigeant de petite entreprise, en particulier dans les domaines économique (significatif) et social (très significatif). Ce dirigeant identifie plutôt mal la portée sociale de l'objectif du développement durable, reliant davantage le concept au traitement des enjeux environnementaux. Cette perception s'accompagne d'ailleurs d'une connaissance plus limitée du DD, d'un intérêt moindre en tant que citoyen et d'un engagement déclaré moins fréquent. Globalement, cette appréhension plus difficile du concept contribue probablement à alimenter la croyance en un objectif davantage adapté aux grandes entreprises.

Tableau 1. Contribution potentielle perçue, engagement, intérêt et connaissance

\begin{tabular}{|l|c|c|l|}
\hline Contribution au domaine économique & $\begin{array}{c}\text { Score moyen } \\
\text { PE (/4) }\end{array}$ & $\begin{array}{c}\text { Score moyen } \\
\text { MGE (/4) }\end{array}$ & $\begin{array}{c}\text { Test } \\
\text { Fischer }\end{array}$ \\
\hline Contribution au domaine social & 2.63 & 2.96 & $0.949^{* * *}$ \\
\hline Contribution au domaine environnemental & 2.63 & 3.25 & $0.998^{* * *}$ \\
\hline Contribution globale au DD & 2.81 & 3.12 & 0.852 \\
\hline Engagement dans une démarche DD & 2.69 & 3.10 & $0.989^{* *}$ \\
\hline Intérêt en tant que citoyen & 2.65 & 3.2 & $0.994^{* * *}$ \\
\hline Degré de connaissance en DD & 3.37 & 3.61 & $0.9686^{* *}$ \\
\hline
\end{tabular}

Tout en considérant moins leur organisation capable de contribuer au DD, les dirigeants de PE attachent autant d'importance que les répondants des MGE aux enjeux environnementaux et attribuent globalement un score d'importance significativement plus élevé aux enjeux intéressant leur personnel. Ce résultat confirme la présomption d'une attention plus soutenue aux salariés sans indiquer un désintérêt plus marqué pour les questions environnementales, ce qui nuance la portée présupposée d'un comportement égocentrique des dirigeants de PE.

Tableau 2. Importance attribuée globalement aux enjeux sociaux et environnementaux

\begin{tabular}{|l|c|c|c|c|}
\hline Domaine du DD & $\begin{array}{c}\text { Score moyen } \\
\text { PE (/4) }\end{array}$ & $\begin{array}{c}\text { Score moyen } \\
\text { MGE (/4) }\end{array}$ & Test F & Test MW \\
\hline Les enjeux sociaux & 3.09 & 2.90 & $0.965^{* *}$ & $0.944^{*}$ \\
\hline Les enjeux environnementaux & 3.16 & 3.26 & 0.504 & 0.289 \\
\hline
\end{tabular}

L'examen du score environnemental par item ne met pas en évidence de spécificités propres à la petite entreprise. Les répondants jugent les enjeux environnementaux tout aussi importants que leurs homologues, qu'il s'agisse de la gestion des déchets, de la réduction des émissions

\footnotetext{
${ }^{3}$ Nous présentons, pour chaque test de comparaison, les scores moyens obtenus ainsi que (1-P), P étant la probabilité de risque attachée à la réfutation de l'hypothèse nulle (absence de différence) avec l'annotation * pour une différence peu significative, ** significative, *** très significative. Les scores sont établis à partir d'une échelle d'intervalle à 4 niveaux, sauf précision contraire.
} 
polluantes ou encore d'une consommation mesurée des ressources énergétiques. En revanche, le dirigeant de PE présente une approche sensiblement différente des enjeux sociaux. Il accorde plus d'importance à des dispositions qui prennent en compte la figure du salarié audelà des domaines traditionnels de la gestion des ressources humaines. Il porte ainsi plus d'intérêt à l'équilibre vie privée-vie professionnelle des salariés, à la variété des tâches accomplies, à la mise en place d'horaires choisis ou encore à la valorisation des efforts. Ces dispositions sont à relier au fonctionnement plus souple des PE, à une moindre spécialisation des tâches en leur sein et rendent compte également d'un effet de proximité. Par ailleurs, bien qu'il place les actions de prévention de la sécurité et de la santé au travail en tête de ses préoccupations, le dirigeant de PE attribue en moyenne à ces enjeux un score plus faible que les répondants des moyennes et grandes entreprises. Au sein des MGE, des relations sociales institutionnalisées (présence éventuelle d'un CHSCT) et un impact économique direct des accidents du travail et des maladies professionnelles pour les entreprises de cette taille contribuent certainement à expliquer cette différence.

Cela étant, les PE ne se différencient pas clairement de leurs homologues de plus grande taille au plan du dialogue et de l'écoute des salariés ou au plan de la participation des salariés aux décisions. Autrement dit, la proximité entre le dirigeant et le personnel n'est pas clairement différenciatrice en la matière, laissant supposer que l'institutionnalisation du dialogue social au sein d'entreprises de plus grande taille compense en partie un déficit de proximité en dirigeant l'attention des managers sur ces aspects.

Tableau 3. Scores d'importance attribués aux enjeux sociaux par item

\begin{tabular}{|l|c|c|c|c|}
\hline Liste des items concernant les enjeux sociaux & PE & MGE & TEST F & TEST MW \\
\hline L'amélioration des conditions de travail & 3.32 & 3.48 & 0.663 & 0.498 \\
\hline Prévention de la santé-sécurité au travail & 3.42 & 3.72 & $0.983^{* *}$ & $0.908^{*}$ \\
\hline Formation des salariés & 3.33 & 3.36 & 0.121 & 0.177 \\
\hline Valorisation des efforts fournis par les salariés & 3.26 & 3.00 & 0.869 & $0.918^{*}$ \\
\hline Dialogue et écoute des salariés & 3.31 & 3.16 & 0.705 & 0.871 \\
\hline Participation des salariés aux décisions & 2.49 & 2.25 & 0.806 & 0.74 \\
\hline Variété des tâches accomplies par les salariés & 3.04 & 2.5 & $0.99^{* *}$ & $0.995^{* * *}$ \\
\hline Mise en place d'horaires choisis pour les salariés & 2.66 & 2.20 & $0.983^{* *}$ & $0.975^{* *}$ \\
\hline Prévention de la discrimination & 3.38 & 3.24 & 0.58 & 0.654 \\
\hline Prise en compte de l'équilibre vie privée/ professionnelle & 3.10 & 2.60 & $0.987^{* *}$ & $0.993^{* * *}$ \\
\hline TIC favorisant le travail à domicile & 2.55 & 2.0 & $0.926^{*}$ & $0.921^{*}$ \\
\hline
\end{tabular}

En ce qui concerne les avantages que les répondants attribuent aux démarches DD, les PE accordent un score inférieur aux MGE pour tous les items envisagés, même si la différence de score global est statistiquement peu significative. Ceci n'est probablement pas étranger au fait qu'elles s'engagent moins fréquemment dans des démarches identifiées comme telles. Par ailleurs, elles hiérarchisent les avantages du DD comme leurs homologues, plaçant les avantages de marché avant les bénéfices d'une réduction des coûts ou d'une meilleure rentabilité des fonds investis. Cela étant, elles considèrent dans une moindre mesure le DD comme un mode de réponse aux attentes de leurs clients. La conformation à des attentes sociétales, codifiées ou non par la loi, reste en tête des avantages perçus, même si l'amélioration de la réputation est significativement plus marquée pour les répondants de MGE, rejoignant en cela la littérature. Relevons que les démarches DD sont appréhendées par les $\mathrm{PE}$ avec moins de force comme la réponse à une attente des collectivités, avec cependant un lien faiblement significatif qui ne permet pas de conclure quant à une moindre ouverture des PE envers la communauté locale. 
Tableau 4. Les avantages perçus réels et potentiels du DD

\begin{tabular}{|l|c|c|c|c|}
\hline Liste des items concernant les avantages du DD & PE & MGE & Test F & Test MW \\
\hline Améliorer la réputation de l'organisation & 3.07 & 3.56 & $0.999^{* * *}$ & $0.946^{*}$ \\
\hline Anticiper et répondre aux attentes réglementaires & 3.05 & 3.36 & $0.906^{*}$ & 0.852 \\
\hline Réduire les coûts & 2.48 & 2.64 & 0.481 & 0.501 \\
\hline Etre en phase avec les valeurs actuelles de la société & 3.11 & 3.24 & 0.514 & 0.300 \\
\hline Trouver de nouveaux débouchés commerciaux & 2.68 & 3.10 & 0.882 & 0.88 \\
\hline Améliorer la rentabilité des fonds investis & 2.41 & 2.65 & 0.682 & 0.696 \\
\hline Conserver ou conquérir un avantage concurrentiel & 2.78 & 3.13 & 0.845 & 0.849 \\
\hline Total score avantages & $\mathbf{2 . 8 0}$ & $\mathbf{3 . 1 0}$ & $\mathbf{0 . 9 4 4} *$ & $\mathbf{0 . 9 1 6}^{*}$ \\
\hline
\end{tabular}

Tableau 5. Le DD comme mode de réponse aux attentes des parties prenantes

\begin{tabular}{|l|c|c|c|c|}
\hline Score d'importance par item & PE & MGE & Test F & Test MW \\
\hline Attente de vos salariés & 2.5 & 2.52 & 0.929 & 0.964 \\
\hline Attente de vos clients ou donneurs d'ordre & 2.65 & 3.12 & $0.017^{* *}$ & $0.057^{* *}$ \\
\hline Attente de vos fournisseurs & 1.96 & 1.96 & 0.963 & 0.852 \\
\hline Attente des collectivités locales & 2.7 & 3.08 & $0.053^{*}$ & 0.105 \\
\hline Attente des investisseurs en capital & 2.14 & 2.45 & 0.215 & 0.170 \\
\hline Attente de vos prêteurs & 1.94 & 2.12 & 0.452 & 0.383 \\
\hline Total score attentes des parties prenantes & $\mathbf{2 . 3 7}$ & $\mathbf{2 . 5 9}$ & $\mathbf{0 . 1 5 5}$ & $\mathbf{0 . 1 5 9}$ \\
\hline
\end{tabular}

En considérant les seules entreprises engagées dans des démarches DD, des différences apparaissent quant aux facteurs moteurs de leur engagement. Si l'idée d'un devoir moral envers les citoyens et la promotion des valeurs organisationnelles constituent les facteurs communément cités en premier par les répondants quelle que soit la taille de l'entreprise, en revanche, les attentes des partenaires financiers, la possibilité d'améliorer la rentabilité financière et dans une moindre mesure, les exigences des partenaires commerciaux représentent des facteurs d'engagement moins marqués au sein des $\mathrm{PE}$. Ces dernières ont donc une approche moins instrumentalisée de leur démarche DD; ceci est également à relier à une indépendance capitalistique plus fréquente permettant de s'affranchir d'une pression des investisseurs sur les politiques.

Tableau 6. Les facteurs favorisant l'engagement en DD

\begin{tabular}{|l|c|c|c|c|}
\hline Liste des items concernant les facteurs d'engagement & PE & MGE & Test F & Test MW \\
\hline Le fait qu'une telle démarche peut améliorer la rentabilité... & 2.66 & 3.04 & $0.974^{* *}$ & $0.903^{*}$ \\
\hline Les exigences de mes partenaires commerciaux... & 2.44 & 2.80 & $0.949^{*}$ & 0.885 \\
\hline Les valeurs promues par mon organisation & 2.96 & 3.2 & 0.732 & 0.576 \\
\hline Les exigences de la règlementation & 2.82 & 3.20 & 0.898 & 0.857 \\
\hline Les attentes des partenaires financiers & 1.81 & 2.32 & $0.978^{* *}$ & $0.984^{* *}$ \\
\hline Les attentes des collectivités locales et des pouvoirs publics & 2.65 & 2.96 & 0.834 & 0.832 \\
\hline ...une telle démarche constitue un devoir moral/les citoyens & 3.41 & 3.36 & 0.241 & 0.192 \\
\hline ...une telle démarche est nécessaire à la survie de l'entreprise & 2.56 & 2.52 & 0.115 & 0.111 \\
\hline TOTAL score facteurs d'engagement & $\mathbf{2 . 6 7}$ & $\mathbf{2 . 9 2}$ & $\mathbf{0 . 9 4 8 *}$ & $\mathbf{0 . 8 9 6}$ \\
\hline
\end{tabular}

Par ailleurs, globalement, les répondants des PE et des MGE ne se distinguent pas quant à l'importance des freins perçus à une démarche DD. Le manque de temps relaté fréquemment (voir par exemple, le baromètre du Crocis) n'est pas davantage perçu comme un frein pour les PE. Ce facteur apparaît d'ailleurs en sixième position pour ces entreprises alors qu'il est cité en deuxième position des freins les plus forts pour les MGE. De même, le déficit de compétences en interne n'est pas davantage considéré comme un frein par les répondants des 
PE. Notons qu'un niveau moindre de connaissance et d'engagement en DD (relevé précédemment), peut conduire le répondant à sous-estimer les compétences dont il a besoin. La méconnaissance des instruments de gestion du DD en tête des freins cités, concerne tout autant les MGE que les PE, confirmant que l'instrument de gestion est considéré comme une voie majeure d'entrée dans le DD, quelle que soit la taille de l'entreprise. Quand on examine l'instrumentation mobilisée par les entreprises engagées, il apparaît que les normes procédurales et les labels ne sont pas moins fréquents au sein des PE, laissant entrevoir une diffusion étendue de ces normes, diffusion reliée à leur valeur institutionnelle. Les PE sont cependant moins concernées par l'élaboration de chartes ou de codes de conduite et fort peu par les instruments de reporting sociétal. La proximité supposée des parties prenantes requiert de fait une communication moins formalisée des principes d'engagement et des résultats.

Le manque d'informations concernant le DD est appréhendé comme un frein plus important pour les répondants des $\mathrm{PE}$. Ce résultat est à rapprocher d'un niveau de connaissance plus faible en la matière (cf infra). De même, l'accès difficile à une offre de services externes peu lisible et l'insuffisance des moyens financiers constituent des freins plus marqués au sein des PE. Notons que les PE engagées en DD de notre échantillon s'adressent moins fréquemment à un consultant externe.

Tableau 7. Les freins à l'engagement de démarches DD

\begin{tabular}{|l|c|c|c|c|}
\hline Liste des items concernant les freins & PE & MGE & Test F & Test MW \\
\hline Un intérêt mineur pour la question & 1.67 & 1.68 & 0.028 & 0.127 \\
\hline Un manque de temps à consacrer à cette question & 2.22 & 2.48 & 0.747 & 0.744 \\
\hline Un manque d'informations sur ce que recouvre le DD & 2.75 & 2.32 & $0.961^{* *}$ & $0.97 * *$ \\
\hline Une méconnaissance des instruments de gestion du DD & 2.85 & 2.68 & 0.581 & 0.637 \\
\hline Une insuffisance de moyens financiers & 2.76 & 2.20 & $0.982^{* *}$ & $0.983^{* *}$ \\
\hline Une telle démarche risque de nuire à la rentabilité ... & 2.06 & 1.8 & 0.751 & 0.65 \\
\hline Des compétences non disponibles/inexistantes en interne & 2.44 & 2.36 & 0.322 & 0.366 \\
\hline Une offre de services externes peu accessible/peu lisible & 2.32 & 1.84 & $0.989 * *$ & $0.967 * *$ \\
\hline Une conjoncture économique défavorable & 2.51 & 2.44 & 0.214 & 0.237 \\
\hline Total Score des freins à l'engagement & 2.39 & 2.20 & 0.862 & 0.856 \\
\hline
\end{tabular}

Tableau 8. L'instrumentation de gestion mobilisée par les entreprises engagées

\begin{tabular}{|l|l|l|}
\hline Instrument concerné & \multicolumn{1}{|c|}{ Test Chi-deux } & Précisions \\
\hline Code de conduite/charte & $0.9383^{*}$ & Moindre recours pour les PE \\
\hline Labels écologiques/normes environnementales & Non significatif & Pas de différence avérée \\
\hline Labels sociaux/normes sociales & Non significatif & Pas de différence avérée \\
\hline Normes en management de la qualité & Non significatif & Pas de différence avérée \\
\hline ACV, bilan carbone, neutralité carbone & $0.9991^{* * *}$ & Moindre recours pour les PE \\
\hline Eco-conception, éco-efficience & Non significatif & Pas de différence avérée \\
\hline Reporting sociétal & $0.9669^{* *}$ & Moindre recours pour les PE \\
\hline
\end{tabular}

\section{Pour conclure : discussion des résultats et limites de l'étude}

En réponse à nos questions de recherche, l'étude quantitative réalisée auprès de 129 entreprises de la région PACA conduit à des résultats contrastés où les «proxémies » évoquées dans la littérature présentent une influence à géométrie variable. Ces résultats ne valident que partiellement l'hypothèse d'une spécificité des PE concernant les pratiques en RSE et DD. En effet, si les répondants des PE envisagent de façon moins évidente une contribution organisationnelle au développement durable, s'ils s'engagent moins dans de telles démarches, ils attachent autant d'importance que les répondants des MGE aux enjeux environnementaux de la durabilité. De même, si les répondants des MGE semblent plus 
sensibles aux incitations externes, ils citent tout autant que les PE des motifs moraux d'engagement, ce qui suggère une corrélation cognitive de leurs motivations instrumentales et morales (Donaldson, 1995). Concernant les relations avec les salariés, l'enquête montre que l'écoute et le dialogue ne sont pas davantage mis en avant dans les PE. La recherche effectuée conduit aussi à rejeter deux arguments couramment évoqués : le manque de temps, qui ne constitue pas un frein spécifique à la $\mathrm{PE}$ et les questions environnementales, qui préoccupent pareillement les PE et les MGE.

En revanche, les résultats obtenus confirment certaines spécificités des PE dans leur appréhension et leurs pratiques en RSE/DD : 1) une prise en compte spécifique des besoins du salarié se traduisant notamment par une attention particulière portée à son équilibre de vie (vie professionnelle/privée) ; 2) une approche moins instrumentalisée du DD et de la RSE ; 3) une évaluation moins évidente des avantages organisationnels inhérents à de telles démarches ; 4) un degré de connaissance moindre du DD, qui constitue l'un des traits majeurs distinguant les $\mathrm{PE}$ et les MGE. Les répondants de PE sont moins en capacité de préciser ce que recouvre le terme de DD. Ce constat explique leur difficulté à cerner les avantages organisationnels du DD. Ils considèrent d'ailleurs le manque d'informations en la matière comme un frein plus décisif que les MGE. De fait, les volontés politiques d'une progression des pratiques responsables au sein des $\mathrm{PE}$ gagnent à s'accompagner d'opérations de sensibilisation et d'information à destination de cette population d'entreprises. L'insuffisance de moyens financiers et la difficulté d'accès à des services externes de conseil en DD sont également soulignées plus fortement, suggérant là encore le rôle que peuvent jouer les pouvoirs publics en mobilisant des financements spécifiques.

Cela étant, les résultats mis en évidence révèlent globalement des liens statistiques relativement faibles. La nécessité d'administrer le questionnaire en face à face en raison de sa longueur, réduit fortement les possibilités de collecte d'observations et accroît le temps nécessaire pour constituer un échantillon convenable. Or, une période de collecte plus longue nous exposait à une affectation temporelle des résultats, d'autant que le management du DD est loin de constituer un domaine stabilisé. A fortiori, si la longueur du questionnaire a l'inconvénient de compliquer son administration, sa richesse permet aussi de mettre en évidence certains résultats inédits.

Par ailleurs, en abordant un thème porteur de jugement de valeur (qui n'est pas désireux de contribuer au développement durable de la planète ? rappelons qu'un seul répondant de notre échantillon se dit non concerné en tant que citoyen), le chercheur expose ses résultats au biais psychologique de désirabilité sociale des répondants. De même, il est probable que les représentants et responsables d'entreprises acceptant de répondre à notre enquête soient a priori, plus intéressés par le DD et la RSE. Ceci implique donc un commentaire prudent sur les résultats globaux obtenus (cf infra, partie 2). Néanmoins, nous pouvons supposer que ces biais affectent indifféremment les PE et les MGE, permettant d'inférer des conclusions de nos observations comparatives. Enfin, bien que les approches quantitatives restent peu répandues et peu recommandées pour explorer l'engagement des PME en durabilité (Spence, 2007 ; Paradas, 2008), elles nous semblent utiles à la mise en perspective des études qualitatives. Ainsi, le critère d'indépendance/dépendance capitalistique pourrait jouer un rôle majeur pour expliquer certains traits de l'engagement en DD des PME, Le Vigoureux (2003) soulignant l'incidence de la structure de propriété des moyennes entreprises sur leurs comportements organisationnels. ${ }^{4}$

\footnotetext{
${ }^{4}$ L'étude de notre échantillon sur la base du critère de dépendance/indépendance capitalistique indique par exemple que la réduction des coûts constitue un avantage plus marqué du point de vue des répondants d'entreprises dépendantes (différence très significative). Cela étant, la taille de notre échantillon ne permet pas à ce jour de tester cette différence au sein des seules $\mathrm{PE}$, objet de cette contribution.
} 


\section{Références bibliographiques}

- ACFCI (2006), «La prise en compte du développement durable et de la responsabilité sociétale de l'entreprise par les PME-PMI», octobre, Chambre de commerce et d'industrie.

- Berger Douce S. (2008), «Rentabilité et pratiques de RSE en milieu PME, premiers résultats d'une étude française », Revue Management et Avenir, ${ }^{\circ} 15$, pp 9-29.

- Bon V. (2007), «Engagement sociétal et visions partenariales de la moyenne entreprise », Revue Management et Avenir, cahier spécial piloté par Luc Boyer et Mathilde Divay, «Vers une théorie de la moyenne entreprise », $N^{\circ} 11$, pp 203-214.

- Bon V. (2011), «L'ISO 26000 : quel apport pour le manager?», Revue Sciences de Gestion, ISEOR, N ${ }^{\circ} 84$, pp 49-63.

- Bon V. et Morlet D. (2011), «D’une conception éthique de la RSE à son cheminement au sein d'une moyenne entreprise », In «Le management par les valeurs », sous la direction de L Bergery, Paris, Lavoisier.

- Bocquet R et Mothe C (2010), «Exploring the relationship between CSR and innovation : a comparison between small and large sized French companies », Revue Sciences de Gestion, n ${ }^{\circ} 0$, pp 101-119.

- Courrent J-M., Torrès O. (2005), "A Proxemic Approach of Small Business: the Case of Business Ethics", 50th International Council of Small Business (ICSB), Washington.

- Crocis-CCIP (2009), «Le développement durable dans les entreprises de la région parisienne-baromètre 2009 », http://www.crocis.ccip.fr

- Donaldson T. (1999), "Making Stakeholder Theory Whole", Academy of Management Review, vol 24, p 237-241.

- Dupuis J-C, Haned N., Le Bas C. (2006), "La responsabilité sociale des entreprises (RSE) en Rhône-Alpes. Premiers résultats d'une enquête auprès des PME régionales", Working paper, Groupe de recherche en Economie et Management, 17 pages.

- Fassin Y., Van Rossem A., Buelens M., (2011), "Small-business owner-managers' perceptions of business ethics and CSR-related concepts", Journal of Business ethics, $\mathrm{n}^{\circ} 98, \mathrm{pp} 425-453$.

- Jenkins H. (2004), «A critical of conventional CSR Theory: an SME perspective », Journal of General Management, vol 29, n 4, pp 37-57.

- Jenkins H. (2009), "A business opportunity model of corporate social responsibility for small- and medium-sized enterprises", Business Ethics: A European Review, vol. 18, $\mathrm{n}^{\circ} 1$, pp. 21-36.

- Julien P-A. et Marchesnay M. (1988), «La petite entreprise : principes d'économie et de gestion », Paris, Vuibert, Collection Gestion.

- Lepoutre J. et Heene A. (2006), «Investigating the impact of firm size on small business social responsibility: a critical review », Journal of Business ethics, $\mathrm{N}^{\circ} 67$, pp 257-273.

- Le Vigoureux F. (2003), "Les moyennes entreprises : questions de gouvernance et d'adossement", Economies et sociétés, $\mathrm{n}^{\circ} 13$, p 923-937.

- Moisdon J-C et alii (1997), "Les instruments de gestion à l'épreuve des organizations, Du mode d'existence des outils de gestion », ouvrage collectif sous la direction J-C. Moidson, Paris, Ed Seli Arslan.

- Moore S.B. et Manring S.L., (2009), "Strategy development in small and mediumsized enterprises for sustainability and increased value creation", Journal of Cleaner Production, $\mathrm{n}^{\circ}$ 17, pp 276-282. 
- Murillo D. et Lozano J.M. (2006), «SMEs and CSR : An approach to CSR in their own words », Journal of Business Ethics, n ${ }^{\circ}$, pp 227-240.

- Paradas A. (2008), "La position des petites entreprises face à la responsabilité sociale", Revue de l'organisation responsable, 2008, ${ }^{\circ} 1$, vol 3, pp 39-52.

- Russo A et Perrini (2010), «Investigating stakeholder theory and social capital : CSR in Large firms and SMEs », Journal of Business ethics, ${ }^{\circ}$ 91, pp 207-221

- Sachs Ignacy (1980), Stratégies de l'écodéveloppement, Paris, Économie et humanisme, Les Éditions ouvrières, 137 pages.

- Sachs Ignacy (1997), L'Écodéveloppement: Stratégies pour le xxie siècle, Paris, Syros, 123 pages.

- Spence L.J. (1999), "Does size matter ? the state of the art in small business ethics", Business ethics : a European review, ${ }^{\circ} 8$, pp 163-174.

- Spence L.J., Schmidpeter R, Habish A (2003), «Assessing social capital : SME enterprises in Germany and the UK », Journal of Business ethics, ${ }^{\circ} 47$, pp 17-29.

- Spence L.J. (2007), «CSR and small business in a European policy context : the five C's of CSR and small business research, agenda 2007 », Business and society review, $\mathrm{n}^{\circ} 112$, pp 533-552.

- Steurer R., Langer M.E., Konrad A., Martinuzzi A. (2005), "Corporations, stakeholders and sustainable development: a theoretical exploration of businesssociety relations", Journal of Business Ethics, $\mathrm{n}^{\circ}$ 61, 2005, pp 263-281.

- Torrès O. (1998), "Vingt cinq ans de recherche en PME : une discipline entre courants et contre courants", in "PME, de nouvelles approches", coordonné par O. Torrès, Economica, pp 17-53.

- Torrès O. (2000), "Du rôle et de l'importance de la proximité dans la spécification de gestion des $P M E "$ ", 3ième congrès international francophone sur la PME, Lille. 